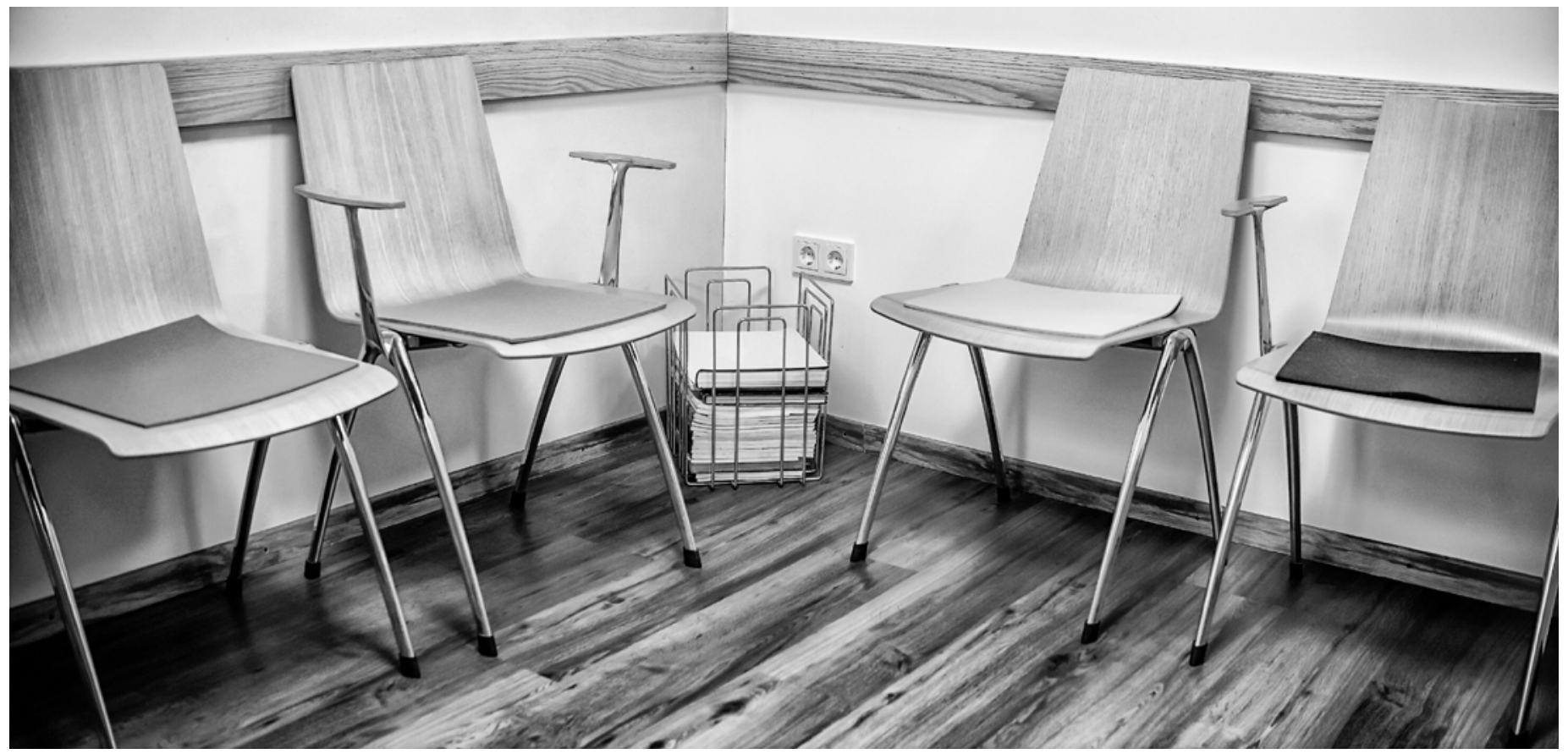

\title{
Tod des Praxisinhabers
}

\section{Katharina Sigrist}

FMH Services (Treuhand)

Der Tod eines nahen Angehörigen ist für die Betroffenen mit schmerzhaftem Abschied verbunden. Zudem steht ein grosser organisatorischer und administrativer Aufwand an. Insbesondere, wenn es sich bei der verstorbenen Person um den Inhaber einer Arztpraxis handelt. Die nachfolgenden Informationen sollen als Hilfestellung in dieser komplexen Situation bei Einzelunternehmern dienen.

\section{Wer muss informiert werden?}

Neben der Trauerarbeit in den ersten Stunden müssen innerhalb von zwei Tagen folgende zuständigen Behörden über den Todesfall informiert werden:

- Zivilstandsamt des Sterbeortes oder

- Bestattungsamt am Wohnort der verstorbenen Person.

Folgende Unterlagen (falls vorhanden) müssen vorgelegt werden:

- Ärztliche Todesbescheinigung oder Todesmeldung

- Personalausweis/Pass/Identitätskarte

- Niederlassungsbewilligung/Aufenthaltsbewilligung (bei Ausländern)

- Schriftenempfangsschein/Familienbüchlein.
Anschliessend stellt das Zivilstandsamt die Todesoder Sterbeurkunde aus.

Selbstverständlich muss das nahe Umfeld der verstorbenen Person informiert werden. Aber auch eine Information an die mit der Berufstätigkeit verbundenen Stellen sollte raschmöglichst erfolgen, um die administrativen Belange zu erleichtern.

- Verbindung der Schweizer Ärztinnen und Ärzte FMH

- Kantonale Ärztegesellschaft und Berufsverbände

- Kantonale Gesundheitsdirektion

- Pensionskasse

- Weitere persönliche Versicherungen

- Vereine und Verbände, in denen der Praxisinhaber Mitglied war 
- Der Notfalldienstbeauftrage (sofern der Notfalldienst des Verstorbenen über die medizinische Gesellschaft organisiert ist). So kann der Notfalldienst rechtzeitig auf einen anderen Kollegen übertragen werden.

- Treuhänder, damit beispielsweise die Löhne und Gehälter der Praxismitarbeitenden weiterhin bearbeitet werden.

Wichtig ist auch die Information an die Patientinnen und Patienten des verstorbenen Arztes. Diese muss durch eine Person erfolgen, welche dem Arztgeheimnis unterliegt (z.B. die medizinische Praxisassistentin). Patientinnen und Patienten müssen persönlich informiert werden, dass sie über ihr Patientendossier verfügen können. Genauere Information zum Vorgehen in den einzelnen Kantonen können über die kantonalen Ärztegesellschaften eingeholt werden.

\section{Erbschein und Testament}

Zur Unterstützung bei den notwendigen Massnahmen zur Erteilung eines Erbscheines und zur Abwicklung des Erbes wird den Hinterbliebenen empfohlen, eine Fachperson (z.B. einen Rechtsanwalt oder Treuhänder) beizuziehen.

Der Erbschein muss bei der zuständigen Behörde beantragt werden. Je nach Kanton ist dies das Erbschaftsamt, die Teilungsbehörde der Gemeinde oder das Regierungsstatthalteramt des Bezirks. Banken und Versicherungen benötigen diese Urkunde als Legitimation zur Weiterführung der Praxis.

Es muss nicht zwingend ein Testament vorhanden sein. Der letzte Wille kann auch in einem Erbvertrag festgehalten sein. Hat der Praxisinhaber aber ein Testament verfasst, muss dieses umgehend der zuständigen Behörde übergeben werden. Es sei darauf hingewiesen, dass sich, wer ein Testament in seinen Besitz nimmt und nicht abliefert oder gar zerstört, strafbar macht. Per Todestag wird durch die zuständige Behörde unter Mithilfe der Erbengemeinschaft ein Erbschaftsinventar erstellt.

\section{Einstellung eines Stellvertreters in der Praxis}

Im besten Fall wurde die Stellvertretung im Todesfall des Praxisinhabers bereits vorab geregelt und ein entsprechender Vertrag liegt vor. In den meisten Fällen obliegt diese Aufgabe jedoch der Erbengemeinschaft. Massgebend für die Regelung der Stellvertretung ist grundsätzlich das kantonale Recht, über welches die kantonalen Ärztegesellschaften wie auch die kantonalen Gesundheitsdirektionen Auskunft geben können.
Es ist zwingend, dass die Stellvertreterregelung schriftlich in einem Vertrag festgehalten wird und die erforderlichen kantonalen Bewilligungen vorliegen.

Die Berufshaftpflichtversicherung des Verstorbenen sollte bis zur Auflösung der Praxis resp. Übergabe an einen Nachfolger weitergeführt werden. Ein Stellvertreter, welcher seine Leistungen über die ZSR-Nummer des verstorbenen Arztes abrechnet, sowie das Praxispersonal bleiben so weiterhin über diesen Vertrag versichert. Nach der Auflösung der Versicherung besteht weiterhin Deckung für Schäden, welche durch den Verstorbenen verursacht wurden, jedoch erst später geltend gemacht werden. Diese Nachrisikodeckung sollte sich über die gesetzlichen Verjährungsfristen erstrecken. Wir empfehlen, diesen Punkt detailliert zu prüfen oder sich durch einen Spezialisten beraten zu lassen.

\section{Erbschaft}

Im Rahmen der Universalsukzession geht die ärztliche Praxis im Todesfall als Vermögenswert des Praxisinhabers auf dessen Erben über. Sobald diese das Erbe annehmen, gehen sowohl Rechte wie auch Pflichten auf sie über. Dies gilt insbesondere für die zehnjährige Aufbewahrungspflicht der Krankengeschichten. Sollten die Erben die Erbschaft ausschlagen, übernehmen die Behörden weitere Schritte.

Wird das Erbe angenommen, wird im Normalfall in einem nächsten Schritt ein Praxisnachfolger gesucht. Dabei ist zu beachten, dass ein Einzelunternehmen nicht als solches weitergegeben werden kann. Es endet mit dem Tod des Einzelunternehmers, und danach wird ein neues Unternehmen gegründet. Materiell erfolgt die ganze oder teilweise Veräusserung des $\mathrm{Ge}$ schäftsbetriebs durch die Übertragung der Aktiven und Passiven und eines allfälligen Goodwills.

Finden die Erben keinen Nachfolger, muss die Praxis liquidiert werden. Hierbei kommen der überlebende Ehepartner, die anderen Erben und die Vermächtnisnehmer - sofern sie die Praxis nicht fortführen - in die steuerliche Privilegierung.

\section{Verträge}

Arbeitsverträge enden nicht mit dem Tod des Praxisinhabers. Die Erben sind allerdings zur Kündigung berechtigt. Liegen keine schriftlichen Arbeitsverträge vor, sind die gesetzlichen Kündigungsfristen einzuhalten. Beim Vorliegen von schriftlichen Arbeitsverträgen sind die darin vereinbarten Kündigungsfristen zu beachten. Von übereilten Kündigungen der Praxismitarbeiter ist allerdings abzuraten, denn einerseits darf der 
für die Übergabe oder Auflösung der Praxis benötigte Zeitaufwand nicht unterschätzt werden. Andererseits besteht die Möglichkeit, dass der Stellvertreter oder gar der Nachfolger die Mitarbeitenden übernehmen möchte. Dies kann positive Auswirkungen auf eine reibungslose Übergabe haben, da die Mitarbeitenden den alltäglichen Betrieb und die Patienten bereits kennen. Das Mietverhältnis der Praxisräume erlischt ebenfalls nicht automatisch mit dem Tod des Praxisinhabers. Die Erben sind berechtigt, den Mietvertrag unter Einhaltung der gesetzlichen Fristen zu kündigen oder das Mietverhältnis fortzusetzen.

Auch sonstige Verträge enden nicht automatisch mit dem Tod des Praxisinhabers, es sei denn, sie sind auf die Person des Inhabers abgeschlossen. Vertragsverhältnisse, die fortbestehen, können von den Erben in der Regel gekündigt werden. Dies betrifft z.B. den Vertrag von Telefon-/Internetanbietern, Wartungsverträge, Software-Abonnementen, Zeitschriften usw. Meist können sie aber auch auf einen allfälligen Nachfolger übertragen werden.

Da der Behandlungsauftrag mit dem Tod des Praxisinhabers endet, müssen laufende Behandlungen abgerechnet werden. Wird die Praxis nicht fortgeführt, sind die Patienten zu informieren, damit sie die Krankengeschichten abholen und sich einen neuen Arzt suchen können. Diese Information kann in einem Anschreiben an die Patienten erfolgen.

\section{Steuern}

Die Erben sind verpflichtet, die für den Erblasser noch abzugebenden Steuererklärungen einzureichen. Dafür ist ein Abschluss (Bilanz/Erfolgsrechnung) per Todestag zu erstellen. In der Steuererklärung der Erben muss das Erbe oder die Beteiligung an einer Erbengemeinschaft erwähnt werden, auch wenn noch keine Aufteilung erfolgt ist.

\section{Fazit}

Neben der Trauerarbeit beim Tod eines Praxisinhabers sind für die Hinterbliebenen viele spezifische administrative Belange zu beachten. Insbesondere die Regelung der Praxisstellvertretung und der Praxisnachfolge kann die Erbengemeinschaft vor grosse Herausforderungen stellen. Es bestehen keine allgemeingültigen Vorgehensweisen, sondern jede Situation muss separat betrachtet und die spezifischen Gegebenheiten berücksichtigt werden. Deshalb ist es sinnvoll, punktuell auf die Unterstützung von Fachpersonen in den jeweiligen Bereichen zurückzugreifen.

\section{Bildnachweis}

(c) Irontrybex | Dreamstime.com 